\title{
Development of hydroxyapatite bone cement for controlled drug release via tetracycline hydrochloride
}

\author{
SAYED MAHMOOD RABIEE \\ Department of Mechanical Engineering, Babol University of Technology, Mazandaran, Babol, Iran
}

MS received 31 May 2008; revised 12 June 2012

\begin{abstract}
The purpose of this work was to study the preparation and characterization of drug-hydroxyapatite cement. The hydroxyapatite (HA) cement has been synthesized by using tricalcium phosphate, calcium carbonate and dicalcium phosphate anhydrous with sodium hydrogen phosphate as liquid phase. The effect of added tetracycline hydrochloride (TCH) as drug on final phases, microstructure, setting behaviour and compressive strength has been studied. The drug release rate was first order within the first day and then was zero order. No obvious difference could be detected in XRD patterns of the TCH-HA cement with various amounts of drug. By increasing the drug concentration, mechanical strength of cement was decreased and its setting time was increased. The results of this study demonstrate the potential of using HA cement as a carrier for drug delivery.
\end{abstract}

Keywords. Calcium phosphate; bone cement; tetracycline hydrochloride; drug delivery.

\section{Introduction}

Calcium phosphates are becoming increasingly popular in the field of biomedical, in particular, dentistry, bone substitute, bone repair materials and drug delivery system (Knabe et al 2000; Komath et al 2000; Rabiee et al 2008). Calcium phosphate cement consists of a powder and a liquid phase. The powder phase contains a mixture of calcium and phosphorous based ingredients mixed with a liquid phase which contains an aqueous medium such as distilled water or aqueous solutions. Mixing of the above components results in precipitation of apatite [AP: $\mathrm{Ca}_{10-x}\left(\mathrm{HPO}_{4}\right)_{x}\left(\mathrm{PO}_{4}\right)_{6-x}$ $(\mathrm{OH})_{2-x}$, where $0 \leq x \leq 2$ ] (Suchaneck and Yoshimura 1998; Ooms et al 2003).

Takagi et al (1998) prepared the first calcium phosphate bone cement that contained tetracalcium phosphate (TTCP) and dicalcium phosphate anhydrous (DCPA) or dicalcium phosphate (DCPD) as the solid phase. After mixing with water, cement forms hydroxyapatite as the only final product (Takagi et al 1998). The advantages of calcium phosphate bone cements are high biocompatibility, bioactivity and osteoconductivity (Yang et al 2002; Fernandez et al 2005). Their serious disadvantage is relatively poor mechanical strength (Ishikawa et al 1995; Fernandez et al 2005). Easy shaping of calcium phosphate bone cements enables using them to fill the bone defects much better than the solid blocks, which are difficult to shape, or the powders/granules, which are difficult to keep in place. The calcium phosphate bone cements may in the future replace PMMA cements as bone/implant fixation if only their mechanical properties can be improved (Okada et al 1999;

(rabiee@ nit.ac.ir)
Fernandez et al 2005). Over the last few years, local delivery systems have attracted much attention due to their efficacy to improve the ingrowths and regeneration of bones and teeth (Peter et al 2005; Sokolova et al 2006). Drugs, such as antibiotics, antitumors and growth factors, have been administered to defect regions to induce therapeutic effects (Lebugle et al 2002; Peter et al 2005). A great deal of effort has been exerted to develop drug carriers, in the form of foams, films and microspheres, aiming at satisfying the requirements, such as safety, greater efficiency, predictable therapeutic response and prolonged release period (Josse et al 2005; Ueno et al 2005; Ginebra et al 2006). The aim was to study the influence of $\mathrm{TCH}$ on the cement properties and drug release ability of synthesized cement. The resulting cements were characterized in terms of setting times and mechanical properties in order to ensure their suitability in skeletal implantation.

\section{Materials and methods}

\subsection{Materials}

The materials used in this study consist of tetracycline hydrochloride (TCH; $\mathrm{C}_{22} \mathrm{H}_{24} \mathrm{~N}_{2} \mathrm{O}_{8} \cdot \mathrm{HCl}$; Sigma-Aldrich), sodium chloride $(\mathrm{NaCl}$; Merck), potassium chloride $(\mathrm{KCl}$; Merck), sodium hydrogen phosphate $\left(\mathrm{Na}_{2} \mathrm{HPO}_{4} \cdot 2 \mathrm{H}_{2} \mathrm{O}\right.$; Merck), monopotassium phosphate $\left(\mathrm{KH}_{2} \mathrm{PO}_{4} \cdot 2 \mathrm{H}_{2} \mathrm{O}\right.$; Merck), calcium carbonate $\left(\mathrm{CaCO}_{3} ;\right.$ Merck), dicalcium phosphate anhydrous $\left(\mathrm{CaHPO}_{4}\right.$; Merck) and $\alpha$-tricalcium phosphate $\left(\alpha\right.$-TCP; $\left.\alpha-\mathrm{Ca}_{3}\left(\mathrm{PO}_{4}\right)_{2}\right) . \alpha$-TCP was synthesized at $1250{ }^{\circ} \mathrm{C}$ by a $2: 1$ molar ratio mixture of dicalcium phosphate anhydrous $\left(\mathrm{CaHPO}_{4}\right)$ and calcium carbonate $\left(\mathrm{CaCO}_{3}\right)$. 


\subsection{Loading of drug in synthesized cement}

The powder phase of the cement was made by mixing $\alpha$ tricalcium phosphate, dicalcium phosphate anhydrous and calcium carbonate. The ratio of starting materials were chosen somehow that the molar ratio of calcium to phosphate $\mathrm{Ca} / \mathrm{P}$ was fixed to be constantly 1.67 , which is the stoichiometric ratio in hydroxyapatite $\left(\mathrm{Ca}_{10}\left(\mathrm{PO}_{4}\right)_{6}(\mathrm{OH})_{2}\right)$. After choosing proper amounts of starting materials, the powder cement was prepared by mixing the starting materials together in a planetary ball mill (Retch PMA, Brinkman, USA) for $6 \mathrm{~h}$. The particle size distribution was determined using a laser particle size analysis (Mastersize 2000, Malvern, USA). The medium particle size, $d_{50}$, reached a limit of about $5 \mu \mathrm{m}$. Different amounts (0, 2, 5, 7 and 10\% w/w) of drug were mixed with powder cement proportionally. The liquid phase of cement was made by dissolving $3 \% \mathrm{NaH}_{2} \mathrm{PO}_{4} \cdot 2 \mathrm{H}_{2} \mathrm{O}$ in double distilled water. The mixture of powder and liquid cement was harmonized and the drug was dispersed into the solidified body uniformly. The cement paste was made and then put into constant temperature incubator $\left(37^{\circ} \mathrm{C}\right.$, relative humidity, $\left.100 \%\right)$.

\subsection{Preparation of simulated body fluid (SBF)}

$8 \mathrm{~g} \mathrm{NaCl}, 0.20 \mathrm{~g} \mathrm{KCl}, 3.63 \mathrm{~g} \mathrm{Na}_{2} \mathrm{HPO}_{4} \cdot 2 \mathrm{H}_{2} \mathrm{O}$ and $0.24 \mathrm{~g}$ $\mathrm{KH}_{2} \mathrm{PO}_{4} \cdot 2 \mathrm{H}_{2} \mathrm{O}$ were dissolved in $800 \mathrm{ml}$ of water, then the $\mathrm{pH}$ value was regulated to 7.40 hydrochloric acid, and at last it was transferred to the vessel with constant volume of $1000 \mathrm{ml}$.

\subsection{In vitro release experiments}

Drug release tests from the cement matrixes were performed at $37^{\circ} \mathrm{C}$ in SBF solution. The cements with different amounts of drug were immersed in $150 \mathrm{ml}$ of dissolution medium in a polyethylene bottle. Aliquots $(2 \mathrm{ml})$ of $150 \mathrm{ml} \mathrm{SBF}$ solution were withdrawn at appropriate intervals from each flask and replaced with fresh SBF. TCH was quantitatively determined in the withdrawn samples by means of a UV-VIS UVIKON 931 Spectrophotometer.

\subsection{Measurement method and instrument}

The product was characterized by X-ray diffraction (Simens, $\mathrm{D}-500, \mathrm{Cu} \mathrm{K} \alpha$ ). Setting times of samples were measured by using a Vicat needle method. Scanning electron microscope (SEM) picture of cements was performed with Stereo Scan 360-Heica Cambridge after coating with gold/palladium. The mechanical strength of the synthesized cements was evaluated as follows: cement paste was packed in a cylindrical plastic mold $(6 \times 12 \mathrm{~mm})$. After setting the cement, it was kept in SBF for 1, 3, 7 and 14 days. The samples were crushed with a cross-head speed of $1 \mathrm{~mm} / \mathrm{min}$ using an Instron Universal Testing Machine (6025) and from there the compressive strength was measured. The values of compressive strength were then averaged for at least six specimens.

\subsection{Statistical analysis}

The differences in setting times and compressive strength between the groups were studied for statistical purposes by one-way ANOVA analysis. Tukey HSD multiple comparison testing was used to determine significance of deviations in the strength of each sample. For all statistical tests, a $p$ value of $<0.05$ was considered to be significant. All statistical analyses were performed with the software program SPSS for Windows, version 9 (SPSS Inc., Chicago, IL, USA).

\section{Results and discussion}

\subsection{Phase and structure of cement}

Mixing of the solid phase was done in a planetary ball mill. Because of hitting the heavy balls with light and small particles of the powder phase, not only the particles were mixed together, but also they got excited and found enough energy to react later with the liquid phase. There are two main types of calcium phosphate compounds depending on the end-product of setting reaction: brushite and hydroxyapatite. Contrary to brushite, which sets in an acidic $\mathrm{pH}$, hydroxyapatite sets in a neutral or basic $\mathrm{pH}$ range. The reaction between liquid and powder phases in cement obeys the acidbase reaction laws (Hench 1991). The results of the X-ray diffraction analysis carried out on the cements indicate the setting reaction of the cement into hydroxyapatite at $24 \mathrm{~h}$ after incubation. Figure 1 shows XRD patterns of cement with $0 \%, 5 \%$ and $10 \%$ of $\mathrm{TCH}$. These patterns showed that the presence of $\mathrm{TCH}$ does not affect significantly the
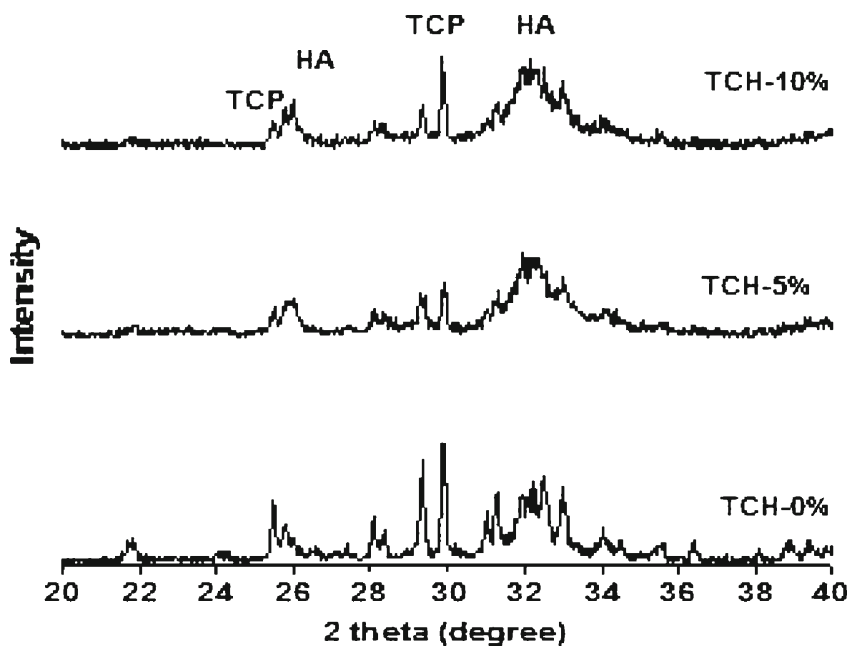

Figure 1. XRD patterns of cement with $0 \%, 5 \%$ and $10 \%$ of $\mathrm{TCH}$ after setting time. 
final phase of the cement that exhibits diffraction reflections characteristic of HA and TCP.

In the drug delivery system, a very important parameter is size distribution of micropores, which in the case of our cement with various $\mathrm{TCH}$ was in the range of $0 \cdot 5-10 \mu \mathrm{m}$. The microstructure affected the setting time and compressive strength greatly. The microstructure before release was observed with SEM, and the result showed that under certain propagation of liquid to powder, the higher the content of the drug, the lower the density of samples, the larger the pore of the product, and the more the large pores. But after a period of release in the simulated body fluid, the microstructure of cement changed noticeably. Figure 2 shows SEM photomicrographs of the cement with $5 \%$ of TCH after 1 week of release. As it is shown in figure $2 \mathrm{a}$, no needle shaped microcrystals of HA was observed in the cement, indicating that these HA crystal are absent before drug release. Micro needle of HA was formed after drug release as depicted in figure $2 \mathrm{~b}$. Those needle-like hydration products were formed by the enrichment of calcium ion and phosphoric ion dissolved in simulated body fluid. This is a slow precipitation process, which is similar to mineralization of human's bone.

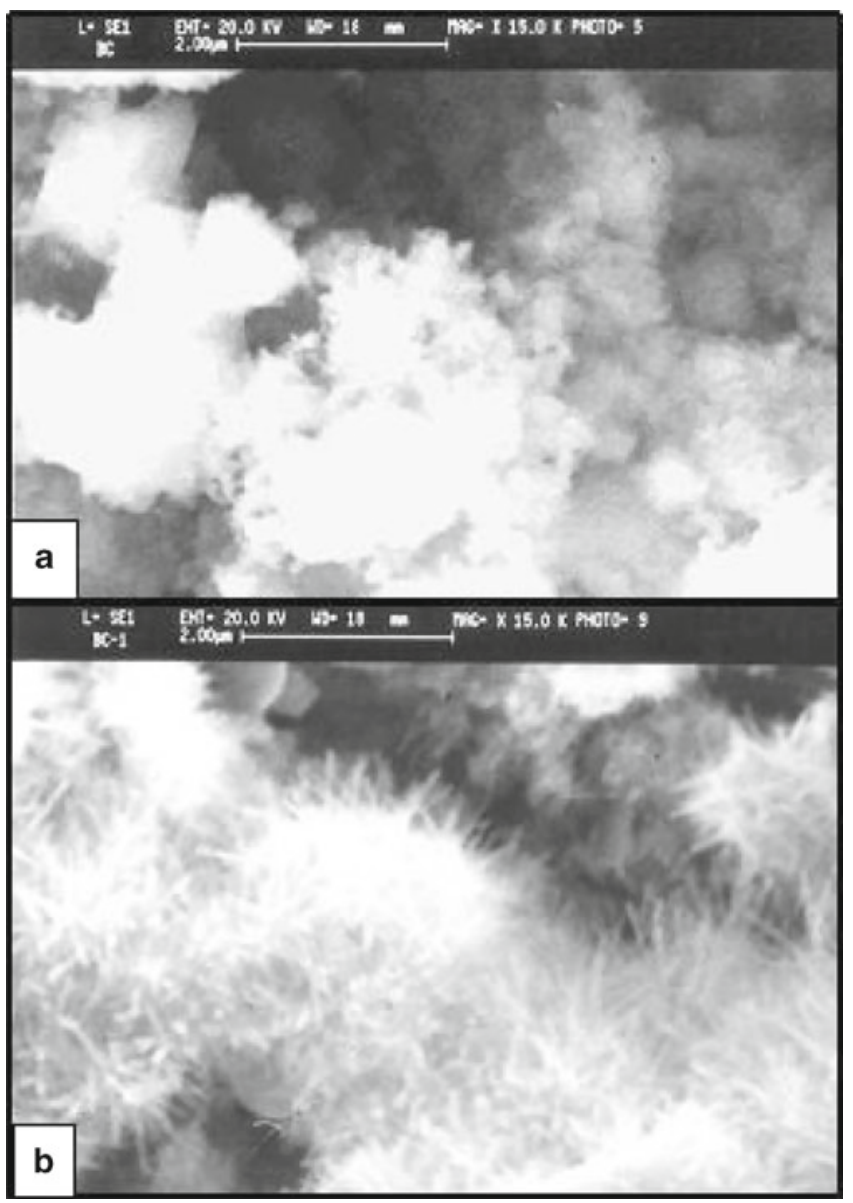

Figure 2. SEM photomicrographs of cement with $5 \%$ of TCH: (a) before release and (b) after release.

\subsection{Kinetics of drug release}

The drug release profile from various cements fabricated is shown in figure 3 . The drug release was first order on the first day and then approached a zero order profile. The amount of $\mathrm{TCH}$ released was increased by raising the concentration of this antibiotic in cement. Similar behaviour was observed for release profile of drug from cements (Schnieders et al 2006). The fast release in the first $24 \mathrm{~h}$ is due to the dissolution of the active ingredient present on the surface of sample. The zero order release profile was attributed to diffusion of drug from cement matrix. The active ingredient dissolves into the liquid within the pores and diffuses into the bulk medium. The first step corresponds to a fast release in the first $24 \mathrm{~h}$. This high percentage could be due to the dissolution of the active ingredient present on the surface of the sample. The second part of curve is nearly linear with a weak slope. It corresponds to a step with nearly constant kinetics of release. This can be explained by a dissolution-diffusion phenomenon. First the active ingredient dissolves into the liquid in the pores and then diffuses from the bulk into the medium. Diffusion of the active ingredient appears to go on until almost its total release.

\subsection{Effect of TCH on setting time}

Setting time is one of the most physical properties of cement, which can judge the feasibility of operation, too long or too short is improper for the operation, and 12-20 min may be feasible (Takagi et al 1998; Barralet et al 2004). The setting time of the cement with various amounts of $\mathrm{TCH}$ are

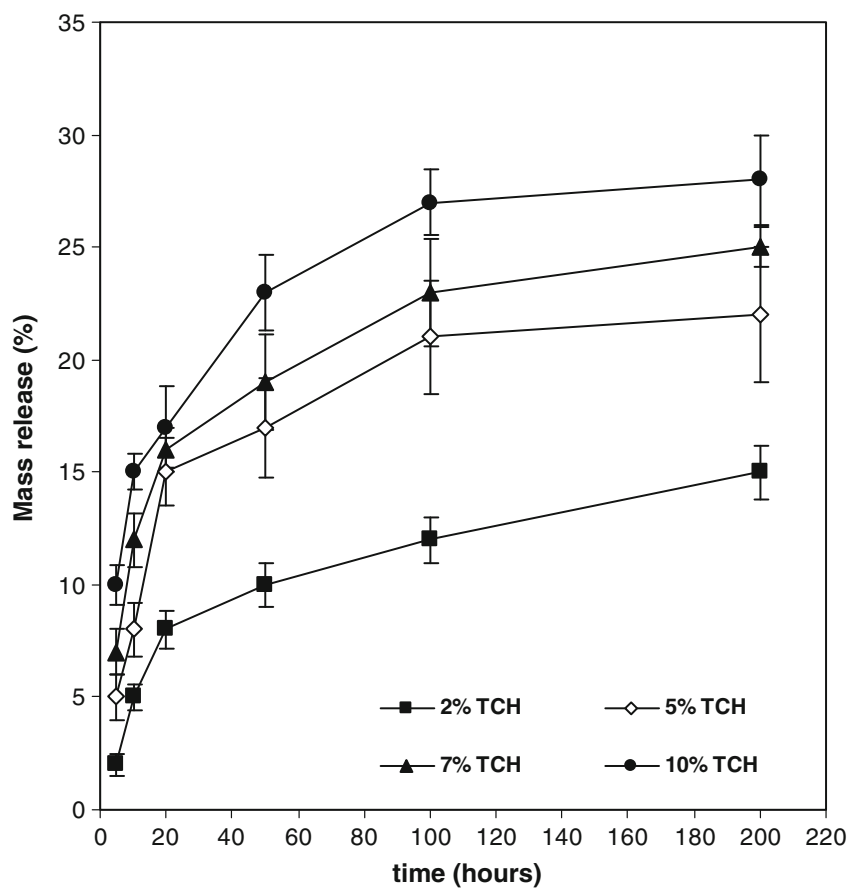

Figure 3. Drug release amount from cements after dissolution test in $\mathrm{SBF}$ at $37^{\circ} \mathrm{C}$ for different times. 
reported in table 1 . The research of TCH-loaded cement has suggested that the setting time significantly increased with increase in the content of the drug. From the angle of clinical application, the content of TCH of $>7 \%$ seems not suitable.

The drug has stable chemical structure and certain polarity. Due to a large number of polar groups, the positive adsorption is produced on the solid-liquid surface, the concentration of drug on the surface is increased, the surface free energy is decreased, and thus the thermodynamic stability of system is improved, which makes the whole system tend to disperse stably and the setting time prolongs with increased content of the drug $(P<0.05)$.

\subsection{Effect of TCH on compressive strength in SBF}

Figure 4 shows compressive strength of the synthesized cement with different contents of drug soaked in the simulated body fluid solution for different times. There are obvious differences in compressive strength before and after the release $(P<0.05)$. It was found that the samples had the highest strength after 7 days of soaking in the solution and then the strength gradually decreased after this time. Increase in the strength of the cements could be attributed to a new calcium phosphate phase crystallization and crystal growth

Table 1. Setting time of cement as a function of $\mathrm{TCH} \%$.

\begin{tabular}{lr}
\hline Content of TCH (\%) & Setting time (min) \\
\hline 0 & $8 \pm 2 \cdot 5$ \\
2 & $13 \cdot 5 \pm 1 \cdot 5$ \\
5 & $17 \pm 2 \cdot 0$ \\
7 & $20 \pm 1 \cdot 5$ \\
10 & $25 \cdot 5 \pm 1 \cdot 5$ \\
\hline
\end{tabular}

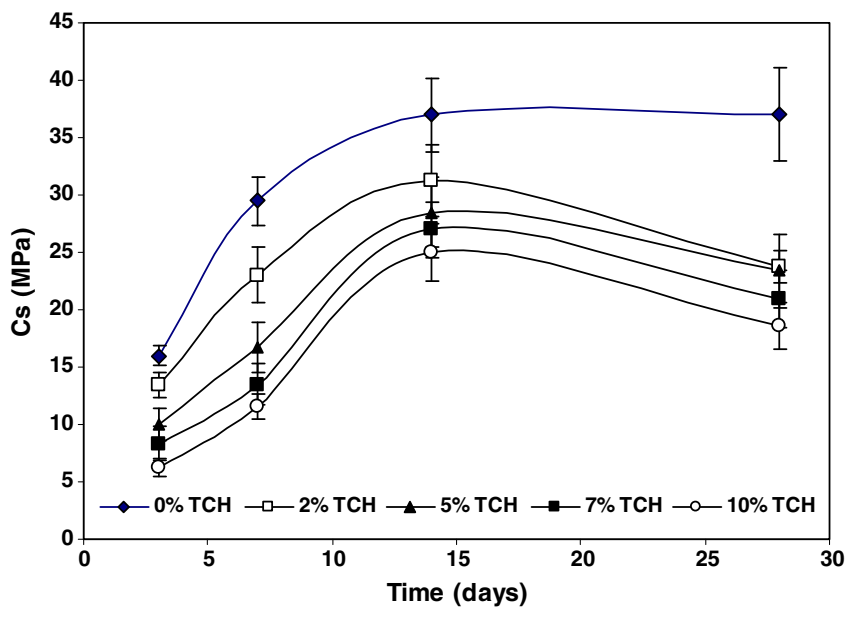

Figure 4. Effect of TCH on compressive strength of cement in SBF solution for different times. within the cement particles and decreased after this time could be due to release of drug and production of pore body $(P<0.05)$.

\section{Conclusions}

The feasibility of a bone cement containing tetracycline hydrochloride was demonstrated in this study. Mixing of the drug with cement did not affect the final products and composition of the hydration products remained unchanged. The study of the release kinetics showed that the drug of cements was released relatively quickly within short periods and then release rate slowed with time. With increase of the content of the drug, the setting time prolonged and the compressive strength decreased. There are obvious differences in compressive strength before and after the release. All of the samples had the highest strength after 7 days of soaking in the simulated body fluid solution and then the strength gradually decreased after this time. Composites made from tetracycline hydrochloride and bone cement could be of interest as a novel drug delivery system in prevention and treatment of bone infections.

\section{References}

Barralet J E, Grover L M and Gbureck U 2004 J. Biomater. 252197 Fernández E, Sarda S and Hamcerencu M 2005 J. Biomater. 262289 Ginebra M P, Traykova T and Planell J A 2006 J. Control. Release 113102

Hench L 1991 J. Am. Ceram. Soc. 741487

Ishikawa K, Miyamoto Y, Kon M, Nagayama M and Asaoka K 1995 J. Biomater. 16527

Josse S et al 2005 J. Biomater. 262073

Knabe C, Driessens F C M, Planell J A and Gildenhar R 2000 J. Biomed. Mater. Res. 52498

Komath M, Varma H K and Ivakumar R 2000 Bull. Mater. Sci. 23 135

Lebugle A, Rodrigues A, Bonnevialle P, Voigt J J, Canal P and Rodriguez F 2002 J. Biomater. 233517

Okada Y, Kawanabe K, Fujita H, Nishio K and Nakamura T 1999 J. Biomed. Mater. Res. 47353

Ooms E M, Wolke J G C and de Heuvel M T U 2003 J. Biomater. 24989

Peter B et al 2005 Bone $\mathbf{3 6} 52$

Rabiee S M, Moztarzadeh F, Solati-Hashjin M and Salimi-Kenari H 2008 Am. Ceram. Soc. Bull. 8743

Schnieders J, Gbureck U, Thull R and Kissel T 2006 J. Biomater. 274239

Sokolova V, Radtke I F, Heumann R and Epple M 2006 J. Biomater. 273147

Suchaneck W and Yoshimura M 1998 J. Mater. Res. 1395

Takagi S, Chow L C and Ishikawa K 1998 J. Biomater. 191593

Ueno Y, Futagawa H, Takagi Y, Ueno A and Mizushima Y 2005 J. Control. Release 10393

Yang Q, Troczynki T and Liu D M 2002 J. Biomater. 232751 